Forschung

\section{Proteine stören die Kommunikation zwischen Bakterien}

Vor äußeren Einflüssen geschützt, können Bakterien in Biofilmen ungestört wachsen und Krankheiten auslösen. Wissenschaftler der Christian-Albrechts-Universität zu Kiel erforschen, wie bereits die Entstehung von Biofilmen verhindert werden kann. Darauf basierend könnten Alternativen zu Antibiotika entwickelt werden, gegen die viele Krankheitserreger häufig bereits resistent sind. Ihre Studienergebnisse veröffentlichten sie Ende Juli in der Fachzeitschrift Frontiers in Microbiology (DOI: 10.3389/fmicb.2016.01098).

Pathogene Bakterien stellen ein besonderes Problem dar, da sie in einem Biofilm nicht mehr durch normale Antibiotikagabe angreifbar sind. „Eine Möglichkeit, Krankheiten zu verhindern, ist Biofilme gar nicht erst entstehen zu lassen“, sagt deshalb R. Schmitz-Streit, Kiel. Um sich auf Oberflächen zu Zellansammlungen zusammenzulagern, müssen Bakterien über Signalmoleküle (sog. Autoinducer) miteinander kommunizieren. Wird diese Kommunikation unterbrochen, kann sich kein Biofilm bilden. Diese Zell-Zell-Kommunikation, das sog. „Quorum sensing“ (QS), kann durch störend eingreifende Biomoleküle („Quorum quenching“-Proteine, QQ) beeinflusst werden. „Proteine können diese Signalmoleküle abbauen oder so modifizieren, dass sie nicht mehr funktionsfähig sind“, erklärt SchmitzStreit. Das kommunikationsstörende Protein QQ-2 zeigte sich in den Untersuchungen als besonders wirksam. „Dieses Protein ist sehr robust und kann viele verschiedene Biofilme verhindern“, erklärt Weiland-Bräuer. Frühere Studien konzentrierten sich eher darauf, eine bestimmte Sprache von Bakterien zu stören. „Das QQ2-Protein ist dagegen auf eine ,Universalsprache‘ ausgerichtet und kann die Kommunikation von verschiedenen Bakterien stören. Es ist also ein ,genereller Störenfried“." Kann die Kommunikation von pathogenen Bakterien bewusst gestört werden, hindert das die Bakterien daran, in Biofilmen zu wachsen und Krankheiten auszulösen.

Nach einer Mitteilung der ChristianAlbrechts-Universität zu Kiel

Diagnostik

\title{
Erfolgsrate der TBNA bei peripheren pulmonalen Läsionen
}

\section{Trotz neuerer Methoden wie dem endobronchialen Ultraschall, der elektromagnetischen Navigation oder der virtuellen Bronchoskopie spielt die transbronchiale Nadelaspirationen (TBNA) für die Diagnose peripherer pulmonaler Läsionen eine wichtige Rolle. Die Studienergebnisse zur diagnostischen Leistungsfähigkeit variieren jedoch deutlich. Um die Beurteilung der Methode zu verbessern, haben M. Mondoni et al. die Genauigkeit der TBNA in einer Metaanalyse analysiert. Eur Respir J 2016; 48: 196-204}

Für die Untersuchung berücksichtigte die italienische Arbeitsgruppe Beobachtungsund Interventionsstudien, in denen ausschließlich die fluoroskopiegestützte TBNA zur Diagnose peripher gelegener Läsionen eingesetzt wurde. Untersuchungen, die u.a. keine gesonderten Ergebnisse für periphere pulmonale Läsionen präsentierten oder deren Patientenpopulationen $<20$ waren, wurden nicht berücksichtigt. Der Beurteilung der methodologischen Studienqualität diente das Quality Assessment of Diagnostic Accuracy Studies (QUADAS-2). Die Urteilsübereinstimmung zwischen den Auswertern betrug 100\%.

Von insgesamt 2089 identifizierten Berichten standen schließlich 18 Studien für die quantitative und qualitative Analyse zur Verfügung. Der zusammengefasste Stichprobenumfang beinhaltete Daten von 1687 Patienten aus 10 Ländern mit einem Durchschnittsalter von 60,4 Jahren. 61\% der Untersuchungen waren prospektive Kohortenstudien, bei 39\% handelte es sich um retrospektive Untersuchungen. In 50\% der Studien waren Patienten mit Verdacht auf oder mit bereits bekannten malignen Lungenläsionen eingeschlossen, bei der anderen Hälfte handelt es sich jeweils um Patienten mit nicht definierten Rundherden.

\section{TBNA besser als TBB \\ $\nabla$}

Die aus allen 18 Studien errechnete diagnostische Ausbeute betrug 0,53. Bei Läsionen mit in der Comptertomografie sichtbaren positiven Bronchuszeichen erreichte die diagnostische Ausbeute einen Wert von 0,70 , ohne Bronchuszeichen waren es 0,51. Wurde die Rapid on-site Evaluation (ROSE) angewandt, betrug der Wert 0,62, ohne ROSE 0,51. Lagen maligne Läsionen vor, errechnete sich eine diagnostische Ausbeute von 0,55, bei benignen Läsionen betrug der Wert 0,17. Während sich bei Läsionen $\leq 3 \mathrm{~cm}$ eine Ausbeute von 0,55 ergab, erreichte sie bei einer Läsionsgröße $>3 \mathrm{~cm} \mathrm{0,81}$. Gegenüber der transbronchialen Biopsie (TBB) war die diagnostische Leistungsfähigkeit der TBNA größer $(0,60$ vs. 0,45$)$.

Fazit
Die diagnostische Leistungsfähigkeit der
TBNA bei peripheren pulmonalen Läsio-
nen beurteilten die Autoren dieser Meta-
analyse insgesamt als akzeptabel, sie war
der TBB überlegen. Prädiktoren wie Bron-
chuszeichen, Malignität, Läsionsgröße
und ROSE-Anwendung erhöhen die diag-
nostische Ausbeute der TBNA. Aufgrund
der errechneten Sensitivität und der Si-
cherheit der TBNA schlagen die Autoren
vor, diese Methode an den Beginn der di-
agnostischen Sequenz zu stellen.

Matthias Manych, Berlin 